\title{
Laparoscopy-assisted uncut Roux-en-Y operation after distal gastrectomy for gastric cancer
}

\author{
Ichiro Uyama, Yoichi Sakurai, Yoshiyuki Komori, Yasuko Nakamura, Mitsutaka Syoji, Syuhei Tonomura, \\ Ikuo Yoshida, Toshiniko Masui, Kazuki Inaba, and Masahiro Ochiai
}

Department of Surgery, Fujita Health University, School of Medicine, 1-98 Dengakugakubo, Kutsukake-cho, Toyoake, Aichi 470-1192, Japan

\begin{abstract}
In order to prevent the Roux stasis syndrome that sometimes follows Roux-en-Y gastrojejunostomy after distal gastrectomy, a new type of reconstruction, called the uncut Roux-enY technique, has been reported. We successfully performed 42 laparoscopy-assisted uncut Roux-en-Y gastrojejunostomies. Here we describe our technique and the initial outcome.
\end{abstract}

Key words Uncut Roux-en-Y gastrojejunostomy . Laparoscopic and laparoscopy-assisted surgery · Distal gastrectomy

\section{Introduction}

Billroth I gastroduodenostomy is the standard reconstruction technique after distal gastrectomy in Japan, whereas Roux-en-Y gastrojejunostomy is now used worldwide for the prevention of alkaline reflux gastritis, esophagitis, dumping syndrome, and carcinogenesis of the gastric remnant [1-3]. However, some patients who receive Roux-en-Y gastrojejunostomy suffer from the Roux stasis syndrome [4]; this reportedly occurs in approximately $30 \%$ of patients who undergo a Roux-en-Y gastrojejunostomy after distal gastrectomy [5]. This syndrome consists of nausea, vomiting, abdominal discomfort, and bloating after meals. In 1988, van Stiegman and Goff [6] reported an alternative method of Roux construction that included performing a Billroth II gastrojejunostomy, occluding the afferent jejunal lumen with linear staples, and diverting pancreaticobiliary secretions through a jejunojejunostomy, to prevent bile reflux gastritis. This new method is referred to as "uncut Roux-en-Y gastrojejunostomy". In 1990, Morrison et al. [7] and in 1992, Miedema and

Offprint requests to: I. Uyama

Received: February 10, 2005 / Accepted: July 13, 2005
Kelly [8], reported that this method preserved gastric emptying and prevented the Roux stasis syndrome.

In Japan, the uncut Roux operation is not yet considered an alternative method. Moreover, the use of the laparoscopic uncut Roux-en-Y gastrojejunostomy after distal gastrectomy has not been reported in the English-language literature. We performed 42 laparoscopic uncut Roux-en-Y gastrojejunostomies for gastric carcinoma. Here we describe the technical details and the initial results of our surgical technique.

\section{Method}

\section{Operating-room setup}

Under general anesthesia, each patient was placed in the supine position with the legs apart. A $20^{\circ}$ head-up tilt was applied in order to prevent the transverse colon or small intestine from visually compromising the operative field. The surgeon stood on the patient's right, with the first assistant on the patient's left and the camera operator between the patient's legs.

\section{Surgical technique}

After pneumoperitoneum was established using the open technique, five ports were placed and a flexible electrolaparoscope was introduced through the infraumbilical port.

The complete uncut Roux-en-Y gastrojejunostomy is illustrated in the schema in Fig. 1. This method is a modified Billroth II operation, which is accomplished by stapling across the afferent limb of the Billroth II gastrojejunostomy in continuity just proximal to the stomach and performing a side-to-side anastomosis for $40 \mathrm{~cm}$ between the afferent limb and the efferent limb, which is designated a "Braun enteroenterostomy". 


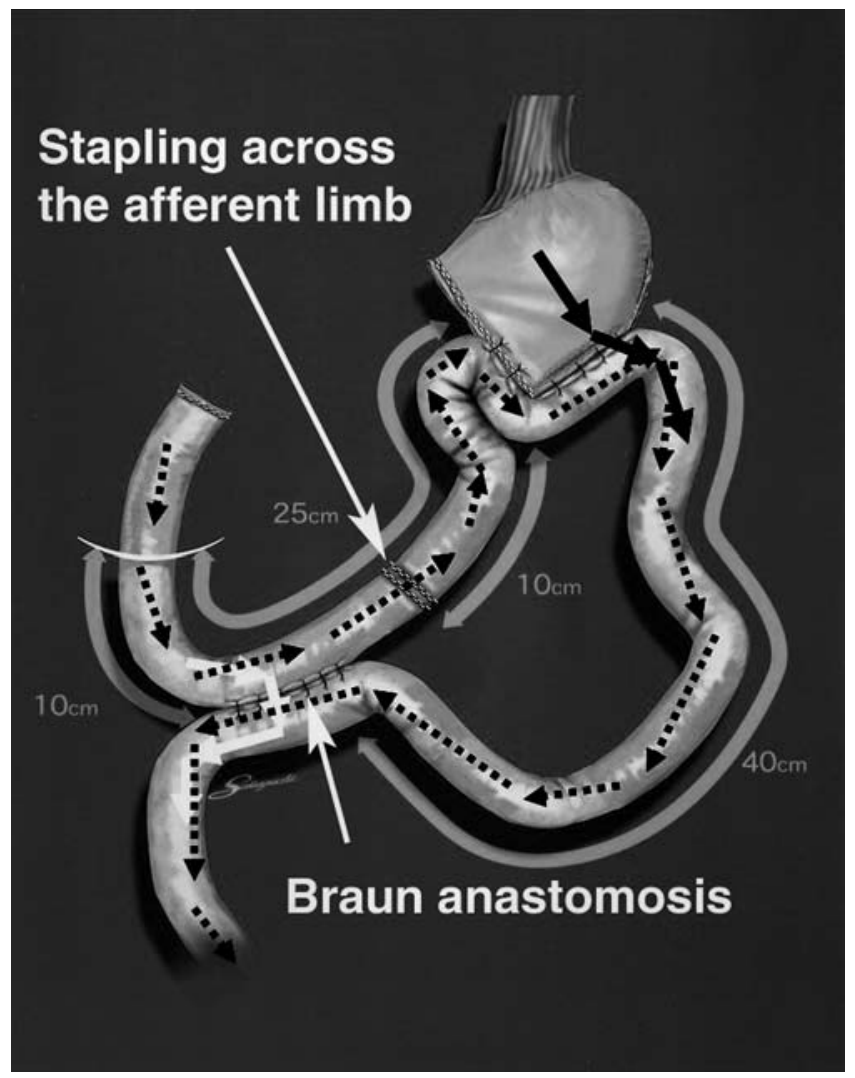

Fig. 1. Schema of the uncut Roux-en-Y operation. The uncut Roux-en-Y operation theoretically maintains more normal neuromotor activity in the Roux limb than the conventional Roux-en-Y method, and minimizes enterogastric reflux. The stapling occlusion of the afferent loop can break down, necessitating conversion of the operation to Billroth II with attendant enterogastric reflux. Solid black arrows indicate the gastric enteric stream. White arrows indicate the biliary enteric stream. Broken black arrows indicate the propagation of enteric pacemaker potentials

We first perform laparoscopic mobilization of the stomach and en-bloc lymph node dissection. Next, a 4$\mathrm{cm}$-long minilaparotomy is made on the upper abdomen, through which the en-bloc-mobilized stomach and lymph nodes are delivered, and the stomach is transected. This procedure thereby allows complete distal gastrectomy with lymph node dissection.

Laparoscopy-assisted reconstruction is then started. First, the transverse colon is retracted cephalad to expose the ligament of Treitz, and the jejunum $25 \mathrm{~cm}$ distal to this ligament is delivered via the minilaparotomy. The position of the gastrojejunostomy, whose length is $4 \mathrm{~cm}$, is determined. Next, a Braun anastomosis is created extracorporeally. We routinely perform the enteroenterostomy by joining the afferent to the efferent limb about 15 and $40 \mathrm{~cm}$, respectively, down from the determined position of the gastrojejunostomy. Next, a stapler without a blade (linear cutter without a knife; Ethicon, Cincinnati, OH, USA) is placed across the

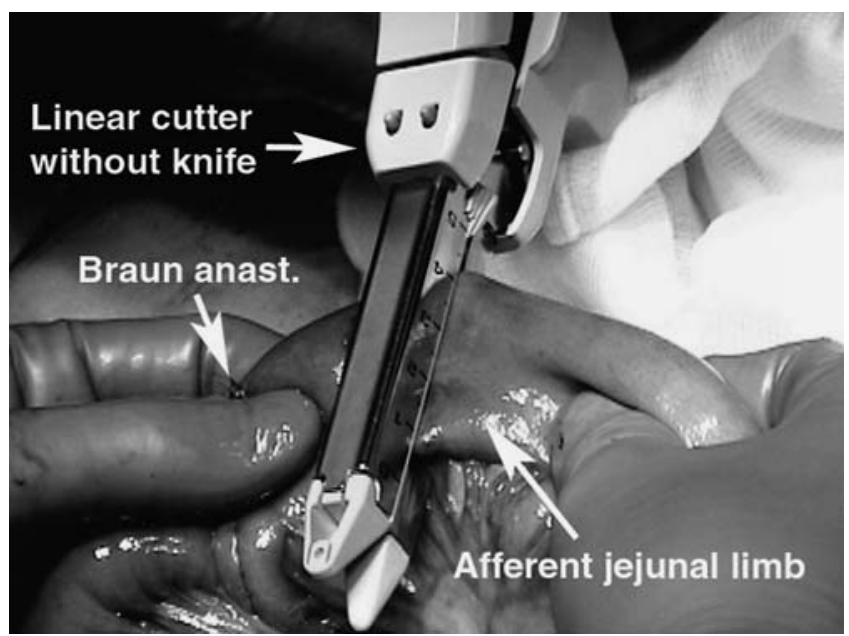

Fig. 2. Stapling across the afferent jejunal limb. A linear cutter without a knife is placed across the afferent jejunal limb just distal to the created Braun anastomosis (Braun anast.)

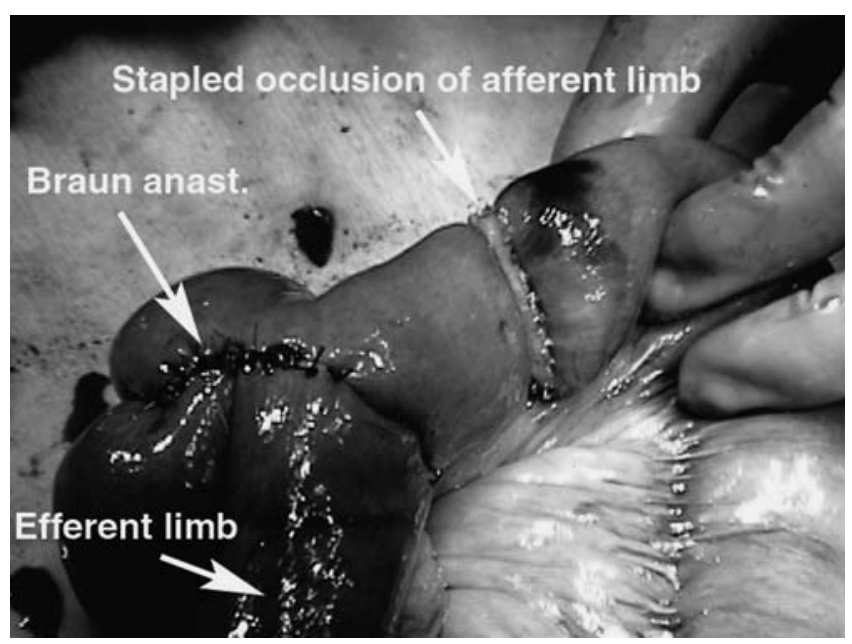

Fig. 3. Stapled occlusion of the afferent jejunal limb. The occlusion of the afferent jejunal lumen without division of the jejunum is performed using the linear cutter without a knife

afferent jejunal limb just distal to the created Braun anastomosis (Fig. 2). This stapler is closed and fired extracorporeally, which enables occlusion of the afferent jejunal lumen without division of the jejunum (Fig. $3)$. Seromuscular sutures are placed on this staple line, and the delivered jejunum is replaced into the abdominal cavity (Fig. 4).

The operation then returns again to a laparoscopic procedure. The gastrojejunostomy is started while observing the created Braun anastomosis and stapling across the jejunum laparoscopically. The corner of the greater curvature of the stomach stump is cut, and a small hole is made on the site of the planned gastrojejunostomy, using Laparosonic Coagulating Shears 


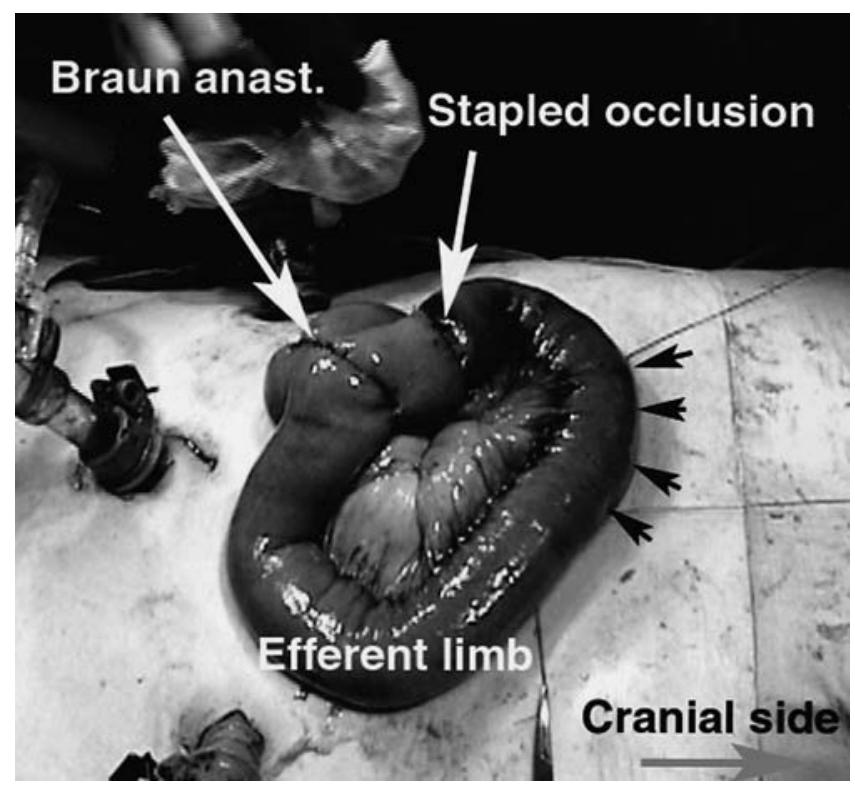

Fig. 4. Completed extracorporeal procedures. The seromuscular sutures are placed on the occlusion staple line and the extracorporeal procedures are completed. Arrowheads show the presumed position of the gastrojejunostomy

(Ethicon). One jaw of the endoscopic linear stapler is inserted into the jejunum and the other into the stomach (Fig. 5A). The device is closed and fired, creating a gastrojejunostomy. The firing of the stapler converts the two holes into one common entry hole, which is closed by a laparoscopic hand-sewn technique (Fig. 5B). Two seromuscular sutures are placed between the afferent loop and the lesser curvature of the gastric remnant to lift up the afferent loop, with the aim of preventing food flowing into the afferent loop. Finally, the seromuscular suture between the stomach and efferent loop is placed on top of the created V-shaped anastomosis, because this is the weakest point, due to the remaining tension. The completed laparoscopic uncut Roux-en-Y anastomosis is shown in Fig. 6.

\section{Results}

From February 2004 to January 2005, we performed laparoscopic uncut Roux-en-Y gastrojejunostomies for gastric cancer in 42 patients who had provided their informed consent after receiving a full explanation of the laparoscopic gastrectomy with the novel reconstruction method.

All procedures were completed without conversion to open surgery. The reconstruction took a mean time of $46 \mathrm{~min}$, which is substantially longer than the corresponding time for an extracorporeal Billroth I gastroduodenostomy $(28 \mathrm{~min})$, but is shorter than the time
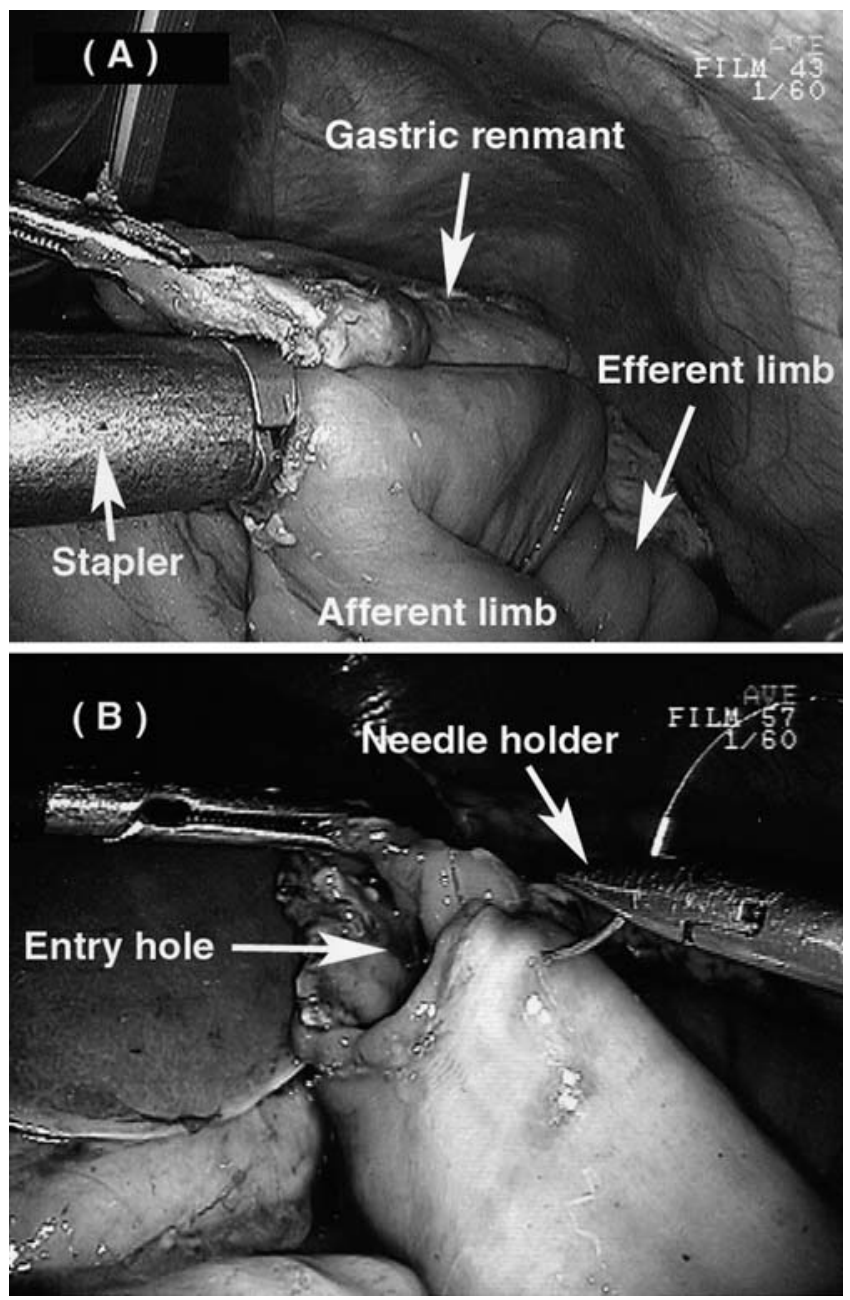

Fig. 5A,B. Laparoscopic gastrojejunostomy. A One jaw of the endoscopic linear stapler is inserted into the jejunum and the other into the stomach. B The created common entry hole is closed by a laparoscopic hand-sewn technique

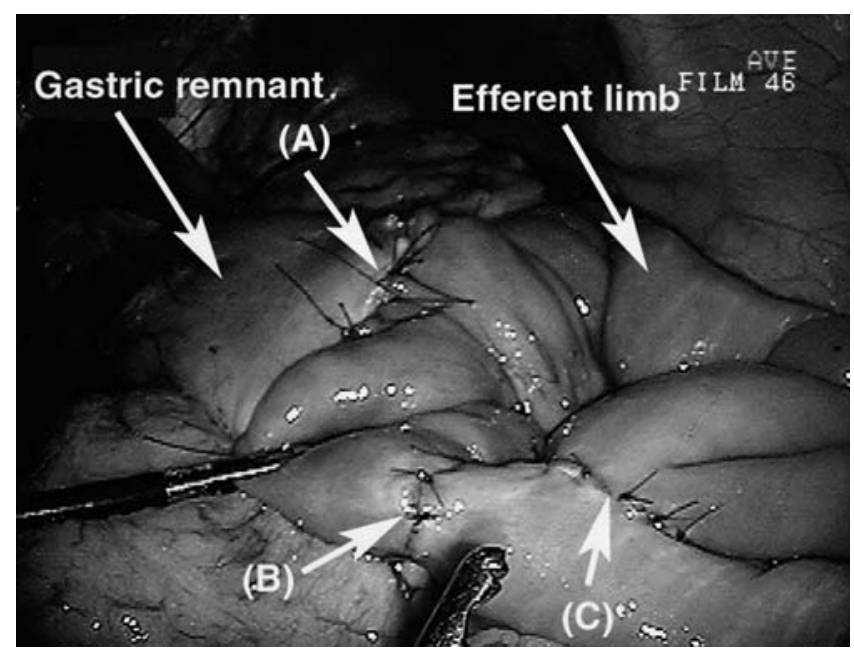

Fig. 6. Completed uncut Roux-en-Y gastrojejunostomy. A Gastrojejunostomy; B stapled occlusion of the afferent jejunal limb; C Braun anastomosis 
taken for a laparoscopy-assisted Roux-en-Y (58 min) or a Billroth II gastrojejunostomy (53 min). There were no instances of anastomotic leakage or stricture.

From November 1997 to January 2005, we performed 54 procedures of laparoscopic or laparoscopy-assisted conventional Roux-en-Y gastrojejunostomy. In the conventional Roux-en-Y gastrojejunostomies, the gastrojejunostomy was created on the greater curvature via an antecolic route, laparoscopically. The difference in the incidence of postoperative gastric stasis between the uncut and conventional Roux operation groups was evaluated using the $\chi^{2}$ test. In all 96 patients (42 with uncut and 54 with conventional Roux operations), the degree of lymph node dissection was less than D2. Postoperative gastric stasis occurred in 6 of the 54 patients (11\%) in the conventional Roux operation group, but in only 2 of the 42 patients $(4.8 \%)$ in the uncut Roux operation group $(P<0.05)$. Although 2 patients suffered from delayed gastric emptying, both of them made a rapid recovery following the suspension of meals for 7 days.

The average follow-up period in the uncut group was 6.6 months (range, 1 to 12 months). None of the 42 patients suffered from reflux esophagitis during the follow up. A postoperative endoscopic examination was performed in 20 patients, and none of these patients experienced bile regurgitation from the jejunum to the gastric remnant. However, we consider that bile reflux in the jejunum to the gastric remnant cannot be diagnosed by incidental endoscopy. We intend assessing the efficacy of hepatobiliary scintigraphy for evaluating bile reflux.

\section{Discussion}

The Roux-en-Y gastrojejunostomy is currently considered a valid reconstruction method after distal gastrectomy for gastric cancer [1-4]. Patients with Roux stasis syndrome [6] present with vomiting, epigastric pain, and weight loss. This syndrome is due to motor incoordination in the intestinal folds, originating from ectopic pacemakers, because the intestine is isolated from its natural pacemakers located in the duodenum, which produces a reflux towards the gastric stump $[7,8]$. In theory, maintaining myoneural continuity between the duodenal pacemaker and the Roux limb can prevent the Roux stasis syndrome. A new type of operation (the uncut Roux-en-Y operation) was reported to maintain this myoneural continuity. This is accomplished by stapling across the afferent limb of the Billroth II gastrojejunostomy in continuity just proximal to the stomach and performing a side-to-side anastomosis between the afferent limb and the efferent limb. Morrison et al. [7] and Miedema and Kelly [8] reported that the staple lines placed across the jejunal wall for the uncut Rouxen-Y operation did not disrupt myoneural continuity to the uncut Roux limb in dogs. Ectopic pacemakers did not appear in the limb, and gastric emptying and Roux limb transit were preserved [7,8]. Noh [9] reported that the uncut Roux-en-Y operation in humans was able to alleviate not only the Roux stasis syndrome but also alkaline reflux gastritis and esophagitis, by preserving the motility of the Roux limb and by diverting duodenal juice from the gastric remnant. We therefore consider the uncut Roux-en-Y operation to be one of the best reconstruction methods after distal gastrectomy.

The use of laparoscopic treatments for gastric cancer is increasing due to the advantages of less invasive surgery [10-13]. The standard reconstruction methods after laparoscopic distal gastrectomy are Billroth I or II, or conventional Roux-en-Y gastrojejunostomy. As far as we are aware, a technique of laparoscopic or laparoscopy-assisted uncut Roux-en-Y gastrojejunostomy has not been reported previously. We performed 42 laparoscopy-assisted uncut Roux-en-Y operations after distal gastrectomy, and the postoperative courses in most of the patients were uneventful. In open surgery, stapling across the afferent is usually started after the accomplishment of the Billroth II gastrojejunostomy. However, in our procedures, first, both the stapling occlusions of the afferent limb and the creation of the Braun enteroenterostomy are performed extracorporeally via a minilaparotomy, and, next, the Billroth II gastrojejunostomy is carried out intracorporeally. We think that it takes a long time to do all of the anastomotic procedures intracorporeally. Hence, it is recommended to perform the stapling occlusion and Braun anastomosis extracorporeally, and the gastrojejunostomy intracorporeally. If the extracorporeal stapling occlusion and Braun anastomosis are performed after intracorporeal gastrojejunostomy, excessive tension may be present at the anastomotic site of the gastrojejunostomy, which can cause leakage of the gastrojejunostomy. We therefore perform the extracorporeal stapling and Braun anastomosis before the intracorporeal gastrojejunostomy.

In intracorporeal gastrojejunostomy, if the enterotomy for insertion of the linear stapler is made on the efferent loop, the procedure of closing the common entry hole - which is created by firing the linear stapler - can cause stricture of the efferent loop and delayed gastric emptying. Therefore, this enterotomy is usually made on the afferent loop.

We usually adopt the antiperistaltic Billroth II gastrojejunostomy in our uncut Roux-en-Y operation. If an isoperistaltic anastomosis and an enterotomy on the afferent loop for is attempted linear stapler insertion, the linear stapler must be inserted through the leftupper trocar, and the insertion of both jaws of the linear 
stapler into the small hole of the afferent loop and gastric remnant is very difficult technically, due to the left subcostal bone hindering the manipulation. However, the antiperistaltic anastomosis enables us to insert the linear stapler via the right-lower trocar and make the enterotomy on the afferent loop. Manipulating the linear stapler via the right-lower trocar is very smooth and safe, hence, our first choice is the antiperistaltic Billroth II gastrojejunostomy. However, if the surgeon judges that an isoperistaltic anastomosis is preferable, because there will be lower tension, and less angulation and undesirable twisting than in the antiperistaltic operation, the isoperistaltic method must be selected. In the isoperistaltic operation, the enterotomy for the insertion of the linear stapler is made on the efferent loop, the linear stapler is inserted through the right-lower trocar, and the created common entry hole has to be closed with a careful and delicate laparoscopic handsewn technique. In six of our patients we adopted the isoperistaltic Billroth II anastomosis, and the postoperative courses in all six were uneventful.

There is one problem with the uncut Roux-en-Y operation: the stapled occlusion of the afferent loop is frequently recanalized, eventually allowing bile access to the gastric remnant [14-16]. In this series we did not experience recanalization of the uncut staple line in the afferent loop, and the incidence of Roux stasis syndrome was lower than that in the conventional Rouxen-Y operation. However, our follow-up period was short and the number of cases was small, and so the postoperative evaluation was inadequate. Therefore, we intend to accumulate further clinical outcomes of this operation.

The laparoscopy-assisted uncut Roux-en-Y operation after distal gastrectomy is both feasible and safe. We consider that our operation may represent the best option for reconstructions after laparoscopic distal gastrectomy.

\section{References}

1. Kelly KA, Becker JM, van Heerden JA. Reconstructive gastric surgery. Br J Surg 1981;68:687-91.

2. Vogel SB, Hocking MP, Woodward ER. Clinical and radionuclide evaluation of Roux-Y diversion for postgastrectomy dumping. Am J Surg 1988;155:57-62.

3. Karlstrom L, Kelly KA. Roux-Y gastrectomy for chronic gastric atony. Am J Surg 1989;157:57-62.

4. Mathias JR, Fernandez A, Aninsky CA, Clench MH, Davis RH. Nausea, vomiting and abdominal pain after Roux-en-Y anastomosis: motility of the jejunal limb. Gastroenterology 1985;88:1017.

5. Gustavsson S, Ilstrup DM, Morrison P, Kelly KA. Roux-Y stasis syndrome after gastrectomy. Am J Surg 1988, 155:490-4.

6. van Stiegman G, Goff JS. An alternative to Roux-en-Y for treatment of bile reflux gastritis. Surg Gynecol Obstet 1988;166:6970 .

7. Morrison P, Miedema BW, Kohler L, Kelly KA. Electrical dysrhythmias in the Roux jejunal limb: cause and treatment. Am J Surg 1990;160:252-6.

8. Miedema BW, Kelly KA. The Roux stasis syndrome. Treatment by pacing and prevention by use of an "uncut" Roux limb. Arch Surg 1992;127:295-300.

9. Noh SM. Improvement of the Roux limb function using a new type of "uncut Roux" limb. Am J Surg 2000;180:37-40.

10. Kitano S, Shiraishi N. Current status of laparoscopic gastrectomy for cancer in Japan. Surg Endosc 2004;18:182-5.

11. Shimizu S, Noshiro H, Nagai E, Uchiyama A, Tanaka M. Laparoscopic gastric surgery in a Japanese institution: analysis of the initial 100 procedures. J Am Coll Surg 2003;197:372-8.

12. Tanimura S, Higashino M, Fukunaga Y, Osugi H. Laparoscopic gastrectomy with regional lymph node dissection for upper gastric cancer. Gastric Cancer 2003;6:64-8.

13. Uyama I, Sugioka A, Fujita J, Komori Y, Matsui H, Soga R, et al. Completely laparoscopic extraperigastric lymph node dissection for gastric malignancies located in the middle or lower third of the stomach. Gastric Cancer 1999;2:186-90.

14. Tu BN, Sarr MG, Kelly KA. Early clinical results with the uncut Roux reconstruction after gastrectomy: limitations of the stapling technique. Am J Surg 1995;170:262-4.

15. Sardinas C, Gattorno F. Evaluation of gastric emptying with the "uncut" Roux en Y technique. Ann Ital Chir 1998;69:41-7.

16. Morton JM, Lucktong TA, Trasti S, Farrell TM. Bovine pericardium buttress limits recanalization of the uncut Roux-en-Y in a porcine model. J Gastrointest Surg 2004;8:127-31. 